\title{
p-Laplacian problems with nonlinearities interacting with the spectrum
}

\author{
Rossella Bartolo, Anna Maria Candela and Addolorata Salvatore
}

Abstract. The aim of this paper is investigating the existence and the multiplicity of weak solutions of the quasilinear elliptic problem

$$
\begin{cases}-\Delta_{p} u=g(x, u) & \text { in } \Omega, \\ u=0 & \text { on } \partial \Omega,\end{cases}
$$

where $1<p<+\infty, \Delta_{p} u=\operatorname{div}\left(|\nabla u|^{p-2} \nabla u\right), \Omega$ is an open bounded domain of $\mathbb{R}^{N}(N \geq 3)$ with smooth boundary $\partial \Omega$ and the nonlinearity $g$ behaves as $u^{p-1}$ at infinity. The main tools of the proof are some abstract critical point theorems in Bartolo et al. (Nonlinear Anal. 7: 981-1012, 1983), but extended to Banach spaces, and two sequences of quasi-eigenvalues for the $p$-Laplacian operator as in Candela and Palmieri (Calc. Var. 34: 495-530, 2009), Li and Zhou (J. Lond. Math. Soc. 65: 123-138, 2002).

Mathematics Subject Classification (2000). 35J92, 58E05, 47J30.

Keywords. Asymptotically $p$-linear problem, $p$-Laplacian, variational methods, linking, pseudo-genus, resonant problem.

\section{Introduction}

Let us consider the following quasilinear elliptic problem

$$
\begin{cases}-\Delta_{p} u=g(x, u) & \text { in } \Omega, \\ u=0 & \text { on } \partial \Omega,\end{cases}
$$

where $1<p<+\infty, \Delta_{p} u=\operatorname{div}\left(|\nabla u|^{p-2} \nabla u\right), \Omega$ is an open bounded domain of $\mathbb{R}^{N}(N \geq 3)$ with smooth boundary $\partial \Omega$ and $g$ is a given real function on $\Omega \times \mathbb{R}$.

Partially supported by M.I.U.R. Research Project PRIN2009 "Metodi Variazionali e Topologici nello Studio di Fenomeni Nonlineari". 
Our aim is obtaining solutions of problem (1.1) when $g$ is asymptotically "linear", i.e. there exists

$$
\lim _{|t| \rightarrow+\infty} \frac{g(x, t)}{|t|^{p-2} t} \in \mathbb{R} \quad \text { uniformly with respect to } x \in \Omega .
$$

So, we consider the interaction of $g$ with the spectrum $\sigma\left(-\Delta_{p}\right)$ of the $p$-Laplacian operator $-\Delta_{p}$ in $W_{0}^{1, p}(\Omega)$, using some sequences of eigenvalues and of quasi-eigenvalues for the $p$-Laplacian.

It is well known that if $p=2$ the spectrum of $-\Delta$ in $H_{0}^{1}(\Omega)$ consists of a diverging sequence $\left(\lambda_{k}\right)_{k}$ of eigenvalues, repeated according to their multiplicity, satisfying $0<\lambda_{1},<\lambda_{2} \leq \ldots \leq \lambda_{k} \leq \ldots$ On the other hand, when $p \neq 2$ the full spectrum of $-\Delta_{p}$ is still unknown, even if various authors have been introducing different characterizations of eigenvalues and definitions of quasi-eigenvalues. Here, we use two sequences of quasi-eigenvalues, denoted by $\left(\eta_{k}\right)_{k}$ and $\left(\nu_{k}\right)_{k}$, following respectively $[10,16]$ (such sequences and their properties are fully described in Sect. 2).

Throughout this paper we suppose that there exist $l_{\infty} \in \mathbb{R}$ and $f$ : $\Omega \times \mathbb{R} \rightarrow \mathbb{R}$ such that

$$
g(x, t)=l_{\infty}|t|^{p-2} t+f(x, t),
$$

so problem (1.1) becomes

$\left(P_{\infty}\right) \quad \begin{cases}-\Delta_{p} u-l_{\infty}|u|^{p-2} u=f(x, u) & \text { in } \Omega, \\ u=0 & \text { on } \partial \Omega .\end{cases}$

Starting from (1.2), let us introduce the following assumptions:

$\left(H_{0}\right) \quad f$ is a Carathéodory function (i.e., $f(\cdot, t)$ is measurable in $\Omega$ for all $t \in \mathbb{R}$ and $f(x, \cdot)$ is continuous in $\mathbb{R}$ for a.e. $x \in \Omega)$ and

$$
\sup _{|t| \leq r}|f(\cdot, t)| \in L^{\infty}(\Omega) \quad \text { for all } \quad r>0
$$

$\left(H_{1}\right)$ there exists

$$
\lim _{|t| \rightarrow+\infty} \frac{f(x, t)}{|t|^{p-2} t}=0 \quad \text { uniformly with respect to a.e. } x \in \Omega ;
$$

$\left(H_{2}\right)$ there exists

$$
\lim _{t \rightarrow 0} \frac{f(x, t)}{|t|^{p-2} t}=l_{0} \in \mathbb{R} \quad \text { uniformly with respect to a.e. } x \in \Omega ;
$$

$\left(H_{3}\right) l_{\infty} \notin \sigma\left(-\Delta_{p}\right)$;

$\left(H_{4}\right)$ there exist $h, k \in \mathbb{N}$, with $k \geq h$, such that

$$
\min \left\{l_{0}+l_{\infty}, l_{\infty}\right\}<\eta_{h} \leq \nu_{k}<\max \left\{l_{0}+l_{\infty}, l_{\infty}\right\}
$$

where $\left(\eta_{h}\right)_{h},\left(\nu_{k}\right)_{k}$ are respectively as in (2.4) and (2.6) below;

$\left(H_{5}\right) f(x, \cdot)$ is odd for a.e. $x \in \Omega$.

We are ready to state our multiplicity result for problem $\left(P_{\infty}\right)$, pointing out that by a solution of $\left(P_{\infty}\right)$ we mean a weak solution, i.e. a function $u \in W_{0}^{1, p}(\Omega)$ satisfying the problem in the sense of distributions. Clearly, $\left(H_{2}\right)$ 
implies that $u=0$ is a solution of the problem; hence we look for non-trivial solutions.

Theorem 1.1. Assume that $\left(H_{0}\right)-\left(H_{5}\right)$ hold. Then, problem $\left(P_{\infty}\right)$ has at least $k-h+1$ distinct pairs of non-trivial solutions.

A result similar to Theorem 1.1 holds also if assumption $\left(H_{3}\right)$, corresponding to the so-called non-resonant case, is avoided, up to assume something stronger on $f$. Here, as in [16], we assume

$\left(H_{3}^{\prime}\right) l_{\infty} \in \sigma\left(-\Delta_{p}\right)$;

$\left(H_{6}\right)$ there exists

$\lim _{|t| \rightarrow+\infty}(f(x, t) t-p F(x, t))=+\infty \quad$ uniformly with respect to a.e. $x \in \Omega$,

with $F(x, t)=\int_{0}^{t} f(x, s) \mathrm{d} s ;$

hence the following result can be stated.

Theorem 1.2. Assume that $\left(H_{0}\right)-\left(H_{2}\right),\left(H_{3}^{\prime}\right)$ and $\left(H_{4}\right)-\left(H_{6}\right)$ hold. Then, problem $\left(P_{\infty}\right)$ has at least $k-h+1$ distinct pairs of non-trivial solutions.

Remark 1.3. The previous multiplicity results hold also if the limit in $\left(H_{2}\right)$ is infinite, i.e. $l_{0} \in\{ \pm \infty\}$ (see Remark 3.2 for more details).

The multiplicity results above are stated in particular under the assumption $\left(H_{5}\right)$, by the way, the existence of at least a non-trivial solution can also be proved in the non-symmetric case. For instance, using the above notations, let us assume that

$\left(H_{7}\right)$ there exists $k \in \mathbb{N}$ such that

$$
\min \left\{l_{0}+l_{\infty}, l_{\infty}\right\}<\eta_{k} \leq \nu_{k}<\max \left\{l_{0}+l_{\infty}, l_{\infty}\right\}
$$

and

$$
\nu_{k-1}<\nu_{k}=\nu_{k+1}=\ldots=\nu_{\bar{k}}<\eta_{\bar{k}+1} \quad \text { for } \quad \bar{k} \in \mathbb{N}, \bar{k} \geq k ;
$$

$\left(H_{8}\right)$ for $k \in \mathbb{N}$ as in $\left(H_{7}\right)$, there exists $\varepsilon_{0}>0$ such that

$$
\left(\nu_{k-1}+\varepsilon_{0}-l_{\infty}\right) \frac{|t|^{p}}{p} \leq F(x, t) \quad \text { for a.e. } x \in \Omega \quad \text { and for all } t \in \mathbb{R} .
$$

Then, the following results can be stated, both in the non-resonant and in the resonant case.

Theorem 1.4. Assume that $\left(H_{0}\right)-\left(H_{3}\right)$ and $\left(H_{7}\right)-\left(H_{8}\right)$ hold. Then, problem $\left(P_{\infty}\right)$ has at least a non-trivial solution.

Theorem 1.5. Assume that $\left(H_{0}\right)-\left(H_{2}\right),\left(H_{3}^{\prime}\right)$ and $\left(H_{6}\right)-\left(H_{8}\right)$ hold. Then, problem $\left(P_{\infty}\right)$ has at least a non-trivial solution.

Remark 1.6. The previous existence results hold also when the limit in $\left(H_{2}\right)$ is infinite (see Remark 4.1 for more details). 
Remark 1.7. By Proposition 2.9 and the monotonicity of $\left(\nu_{k}\right)_{k}$ (see Sect. 2), we have that $\eta_{h} \leq \nu_{k}$ for $k \geq h \geq 1$; thus this inequality is not an assumption, even if it appears in $\left(H_{4}\right)$ and $\left(H_{7}\right)$. Moreover, $\left(H_{1}\right),\left(H_{2}\right)$ and $\left(H_{8}\right)$ imply that $\nu_{k-1}<\min \left\{l_{0}+l_{\infty}, l_{\infty}\right\}$, hence, without loss of generality, in $\left(H_{7}\right)$ we assume that $\nu_{k}$ is the smallest quasi-eigenvalue such that $\nu_{k}>\min \left\{l_{0}+l_{\infty}, l_{\infty}\right\}>\nu_{k-1}$.

When $p=2$ problem $\left(P_{\infty}\right)$ is known as the asymptotically linear one and has been widely investigated (see $[1,5]$ and references therein). In this case $\nu_{k}=\eta_{k}=\lambda_{k}$ for all $k \in \mathbb{N}$ (see Sect. 2), so our results reduce to classical ones or some extensions of them (see also [6, Theorem 3.1], [16, Theorem 1.2]).

In spite of the large amount of papers dealing with this kind of nonlinearities in the semilinear case, only a few results have been obtained when $p \neq 2$. Namely, some existence results can be found in $[3,4,9,12,15,19,20]$. In particular, in [15, Theorem 1.1] the existence of a non-trivial positive solution is obtained for $l_{0}+l_{\infty}=0$ and $l_{\infty}>\mu_{1}$ (see (2.1)), both in the non-resonant and in the resonant case. On the other hand, under some additional conditions on the nonlinearity $f$ near 0 , Liu and Li find non-zero solutions via Morse theory (cf. [19, Theorems 1.2 and 1.3]). Moreover, under assumptions comparable with ours, in [20, Theorem 1.1] Perera and Szulkin use a suitable notion of linking for finding a solution if $l_{0}+l_{\infty}, l_{\infty} \notin \sigma\left(-\Delta_{p}\right)$.

For what concerns multiple solutions, we are aware only of two results. More precisely, in [16] Li and Zhou use the Symmetric Mountain Pass Theorem when the Cerami compactness condition holds (cf. Definition 2.1), proving a multiplicity result if $l_{0}+l_{\infty}=0$; moreover, under further assumptions, they consider both the resonant case and the circumstance $l_{\infty}=+\infty$. At least if $l_{\infty} \notin \sigma\left(-\Delta_{p}\right)$, their result is improved in [20, Theorem 1.2], where it is allowed that $l_{0}+l_{\infty} \neq 0$, even if it is required that $l_{0}+l_{\infty} \notin \sigma\left(-\Delta_{p}\right)$.

Our approach differs from that in [20] since, following [5], we use the pseudo-index theory related to the Krasnoselskii genus (cf. [7] and here Sect. 2), although in [5] only Hilbert spaces are considered and the nonlinearity interacts with the completely known spectrum of $-\Delta$ in $H_{0}^{1}(\Omega)$. On the other hand, in [20] the authors construct a sequence of eigenvalues (see Sect. 2) and use the pseudo-index theory related to the cohomological index of Fadell and Rabinowitz [13].

This paper is organized as follows: in Sect. 2 we define the sequences of quasi-eigenvalues $\left(\eta_{k}\right)_{k}$ and $\left(\nu_{k}\right)_{k}$ which appear in the hypotheses, then we recall their fundamental properties and establish a relation between them (Proposition 2.9). Moreover in our setting we present an abstract critical point theorem as stated in [5] (Theorem 2.6) and we prove an estimate from below for the genus, involving the dimension and the codimension of some subspaces of a Banach space (Theorem 2.7). In Sect. 3 we prove Theorems 1.1 and 1.2; finally, in Sect. 4 we prove Theorems 1.4 and 1.5.

Notations. Throughout this paper we denote by $\left(X,\|\cdot\|_{X}\right)$ a Banach space, by $\left(X^{\prime},\|\cdot\|_{X^{\prime}}\right)$ its dual, by $I$ a $C^{1}$ functional on $X$ and by

- $I^{b}=\{u \in X: I(u) \leq b\}$ the sublevel of $I$ corresponding to $b \in \mathbb{R}$ and $I_{b}=\{u \in X: I(u) \geq b\}$ the superlevel of $I$ corresponding to $b \in \mathbb{R}$; 
- $K_{c}=\{u \in X: I(u)=c, \mathrm{~d} I(u)=0\}$ the set of the critical points of $I$ in $X$ at the critical level $c \in \mathbb{R}$.

Furthermore, let us denote by

- $\mathbb{N}=\{1,2, \ldots\}$;

- $|\cdot|_{s}$ the usual norm in the Lebesgue space $L^{s}(\Omega), 1 \leq s \leq+\infty$;

- $\|\cdot\|$ the norm in $W_{0}^{1, p}(\Omega)$, i.e. $\|u\|=|\nabla u|_{p}$ for all $u \in W_{0}^{1, p}(\Omega)$;

- $B_{R}=\left\{u \in W_{0}^{1, p}(\Omega):\|u\|<R\right\}, S_{R}=\left\{u \in W_{0}^{1, p}(\Omega):\|u\|=R\right\}$ for any $R>0$;

- $p^{*}=\frac{p N}{N-p}$ the critical exponent for the Sobolev embeddings of $W_{0}^{1, p}(\Omega)$ if $p \in] 1, N\left[, p^{*}=+\infty\right.$ otherwise.

\section{Preliminary material and variational tools}

As we have pointed out in Sect. 1, the spectral properties of the $p$-Laplacian in $W_{0}^{1, p}(\Omega)$ are still mostly unknown. For example, two unbounded and increasing sequences of eigenvalues $\left(\mu_{k}\right)_{k}$ and $\left(\mu_{k}^{\prime}\right)_{k}$, with $\mu_{k}^{\prime} \geq \mu_{k}$, have been constructed in [14] (see also [2]) and [20], using respectively the Krasnoselskii genus as in [21, Chapter 7] and the cohomological index of Fadell and Rabinowitz introduced in [13]. Anyway, it is not known whether they cover the whole spectrum $\sigma\left(-\Delta_{p}\right)$ when $N \geq 2$, but the first eigenvalue $\mu_{1}$ and its properties have been exploited and it is characterized as

$$
\mu_{1}=\inf _{u \in W_{0}^{1, p}(\Omega) \backslash\{0\}} \frac{\int_{\Omega}|\nabla u|^{p} \mathrm{~d} x}{\int_{\Omega}|u|^{p} \mathrm{~d} x},
$$

it is positive, simple, isolated and has a unique positive eigenfunction $\varphi_{1}$ such that $\left|\varphi_{1}\right|_{p}=1$ (cf. e.g. [17]). With respect to the semilinear case, the drawback in using eigenvalues is that, for the Banach space $W_{0}^{1, p}(\Omega)$, their use does not provide a decomposition having properties similar to that of the Hilbert space $H_{0}^{1}(\Omega)$ by means of the eigenfunctions of $-\Delta$ on $\Omega$ with null homogeneous Dirichlet data.

As we shall see in the proofs of our main results, the definition of the quasi-eigenvalues proposed in [10] fits in with our purposes as a suitable decomposition of the Sobolev space $W_{0}^{1, p}(\Omega)$ can be introduced, so that it turns out to be the classical one for $p=2$.

In order to present this definition, firstly let us recall that if $V \subseteq X$ is a closed subspace of a Banach space $X$, a subspace $W \subseteq X$ is a topological complement of $V$, briefly $X=V \oplus W$, if $W$ is closed and every $x \in X$ can be uniquely written as $v+w$, with $v \in V$ and $w \in W$; furthermore the projection operators onto $V$ and $W$ are (linear and) continuous, hence there exists $L=L(V, W)>0$ such that

$$
\|v\|+\|w\| \leq L\|v+w\|
$$

(see e.g. [8, p. 38]). When $X=V \oplus W$ and $V$ has finite dimension, we say that $W$ has finite codimension, with $\operatorname{codim} W=\operatorname{dim} V$. 
In [10, Sect. 5], starting from $\eta_{1}=\mu_{1}$, it is shown the existence of an increasing diverging sequence $\left(\eta_{h}\right)_{h}$ of positive real numbers, with corresponding functions $\left(\psi_{h}\right)_{h}$ such that $\psi_{1} \equiv \varphi_{1}$ and $\psi_{i} \neq \psi_{j}$ if $i \neq j$. They generate the whole space $W_{0}^{1, p}(\Omega)$ and are such that

$$
W_{0}^{1, p}(\Omega)=V_{h} \oplus W_{h} \quad \text { for all } h \in \mathbb{N},
$$

where $V_{h}=\operatorname{span}\left\{\psi_{1}, \ldots, \psi_{h}\right\}$ and its complement $W_{h}$ can be explicitely described.

Remarkably for all $h \in \mathbb{N}$ on the infinite dimensional subspace $W_{h}$ the following inequality holds:

$$
\eta_{h+1} \int_{\Omega}|w|^{p} \mathrm{~d} x \leq \int_{\Omega}|\nabla w|^{p} \mathrm{~d} x \quad \text { for all } \quad w \in W_{h}
$$

(cf. [10, Lemma 5.4]).

Unluckily, we don't know whether, by making use of this sequence of quasi-eigenvalues, a reversed inequality holds on finite dimensional subspaces. Then, we require a different setting and take advantage of another sequence, introduced in [16], for obtaining some existence and multiplicity results on a class of $p$-Laplacian problems.

More precisely, for all $k \in \mathbb{N}$ we consider

$$
\mathbb{W}_{k}=\left\{V: V \text { is a subspace of } W_{0}^{1, p}(\Omega), \varphi_{1} \in V \text { and } \operatorname{dim} V \geq k\right\}
$$

and

$$
\nu_{k}=\inf _{V \in \mathbb{W}_{k}} \sup _{u \in V \backslash\{0\}} \frac{\int_{\Omega}|\nabla u|^{p} \mathrm{~d} x}{\int_{\Omega}|u|^{p} \mathrm{~d} x} .
$$

The main properties of $\left(\nu_{k}\right)_{k}$ are the following: $\nu_{1}=\mu_{1},\left(\nu_{k}\right)_{k}$ is an increasing diverging sequence and, if $p=2$, it agrees with $\left(\lambda_{k}\right)_{k}$ (cf. [16, Appendix]).

In Sect. 3 we shall see that problem $\left(P_{\infty}\right)$ has a variational structure, thus next we present the variational framework we are going to use. To this aim we need some abstract tools, so from now till the end of this section we consider a real Banach space $\left(X,\|\cdot\|_{X}\right)$ and a $C^{1}$ functional $I: X \rightarrow \mathbb{R}$.

Firstly, we recall the so-called Cerami's variant of the Palais-Smale condition; even if it is a condition weaker than the classical one, it is enough in order to state a Deformation Theorem and some critical point theorems (cf. [5]).

Definition 2.1. The functional I satisfies the Cerami's variant of the PalaisSmale condition, briefly $C P S$, at level $c(c \in \mathbb{R})$, if any sequence $\left(u_{n}\right)_{n} \subseteq X$ such that

$$
\lim _{n \rightarrow+\infty} I\left(u_{n}\right)=c \quad \text { and } \quad \lim _{n \rightarrow+\infty}\left\|\mathrm{d} I\left(u_{n}\right)\right\|_{X^{\prime}}\left(1+\left\|u_{n}\right\|_{X}\right)=0
$$

converges in $X$, up to subsequences. In general, if $-\infty \leq a<b \leq+\infty, I$ satisfies $(C P S)$ in $] a, b[$ if so is at each level $c \in] a, b[$.

In order to state a classic existence critical point theorem, we recall that, taking a subspace $Y$ of $X$, if $S \subseteq X$ is a closed subset of $X$ and $Q \subseteq Y$ has boundary $\partial Q$ with respect to $Y$, then $S$ and $\partial Q$ link if $S \cap \partial Q=\emptyset$ and $\phi(Q) \cap S \neq \emptyset$, for any $\phi \in C(X, X)$ such that $\left.\phi\right|_{\partial Q}=\mathrm{id}$. 
For further use, we recall two examples of linking sets (cf. [21, Examples 5.22 and 5.26] and also [5, Propositions 2.1 and 2.2] in the case of an Hilbert space).

Example 2.2. Let $V, W$ be two closed subspaces of $X$ such that $X=V \oplus W$ and $\operatorname{dim} V<+\infty$. Then, setting $Q=V \cap B_{R}$ for $R>0$ and $S=W, \partial Q$ and $S$ link.

Example 2.3. Let $V, W$ be two closed subspaces of $X$ such that $X=V \oplus W$, $\operatorname{dim} V<+\infty$, and $e \in W$ with $\|e\|=1$. If $R_{1}, R_{2}, \rho>0$ and

$$
S=S_{\rho} \cap W, \quad Q=\left(B_{R_{2}} \cap V\right) \oplus\left\{t e: t \in\left[0, R_{1}\right]\right\}, \quad Y=V \oplus \operatorname{span}\{e\},
$$

then $S$ and $\partial Q$ link whenever $R_{1}>\rho$.

The following Linking Theorem holds (see [5, Theorem 2.3] or [24, Theorem 2.12]).

Theorem 2.4. Consider $a, b, \alpha, \beta \in \overline{\mathbb{R}}=\mathbb{R} \cup\{ \pm \infty\}$ such that $-\infty \leq a<\alpha<$ $\beta<b \leq+\infty$. Assume that:

(i) the functional I satisfies $(C P S)$ in $] a, b[$;

(ii) there exist a closed $S \subseteq X$ and $Q \subseteq Y$, being $Y$ a subspace of $X$, with boundary $\partial Q$ in $Y$, satisfying:

(a) $I(u) \leq \alpha$ for all $u \in \partial Q$ and $I(u) \geq \beta$ for all $u \in S$;

(b) $S$ and $\partial Q$ link;

(c) $\sup _{u \in Q} I(u)<+\infty$.

Then, there exists a critical level $c$ of I given by

$$
c=\inf _{\phi \in \Gamma} \sup _{u \in Q} I(\phi(u)), \quad \text { with } \quad \beta \leq c \leq \sup _{u \in Q} I(u),
$$

where $\Gamma=\left\{\phi \in C(X, X):\left.\phi\right|_{\partial Q}=\mathrm{id}\right\}$.

Besides some existence critical point theorems, sharper multiplicity results can be stated when one deals with symmetric functionals on Hilbert spaces (see e.g. $[5,21]$ ). In this section we rewrite [5, Theorem 2.9] on Banach spaces where the index theory related to the genus acts. As in [5] the proof is based on the use of a pseudo-index theory, so, before introducing such a definition, we recall some notions of the index theory on Banach spaces $X$ for an even functional with symmetry group $\mathbb{Z}_{2}=\{$ id, -id $\}$ (cf. e.g. [22]).

Define

$$
\begin{aligned}
\Sigma=\Sigma(X)=\{A \subseteq X: A \text { closed and symmetric w.r.t. the origin, } \\
\text { i.e. }-u \in A \text { if } u \in A\}
\end{aligned}
$$

and

$$
\mathcal{H}=\{h \in C(X, X): h \text { odd }\} .
$$

Taking $A \in \Sigma, A \neq \emptyset$, the genus of $A$ is $\gamma(A)=\inf \left\{k \in \mathbb{N}: \exists \psi \in C\left(A, \mathbb{R}^{k} \backslash\{0\}\right)\right.$ s.t. $\psi(-u)=-\psi(u)$ for all $\left.u \in A\right\}$, if such an infimum exists, otherwise $\gamma(A)=+\infty$. Assume $\gamma(\emptyset)=0$. 
The index theory $(\Sigma, \mathcal{H}, \gamma)$ related to $\mathbb{Z}_{2}$ is also called genus (for more details we refer to [22, Sect. 1] and [23, Sect. II.5]). For further use we recall below its main properties: for all $A, B \in \Sigma$

$\left(i_{1}\right) \gamma(A)=0 \Leftrightarrow A=\emptyset$;

$\left(i_{2}\right) \quad A \subseteq B \Rightarrow \gamma(A) \leq \gamma(B)$ (monotonicity property);

$\left(i_{3}\right) \gamma(A \cup B) \leq \gamma(A)+\gamma(B)$ (subadditivity property);

$\left(i_{4}\right) \gamma(A) \leq \gamma(\overline{h(A)})$ for all $h \in \mathcal{H}$ (supervariancy property);

$\left(i_{5}\right)$ if $A$ is compact, there exists $\delta>0$ such that $\gamma\left(N_{\delta}(A)\right)=\gamma(A)$, where $N_{\delta}(A)=\{x \in X: d(x, A) \leq \delta\}$ is the closed $\delta$-neighbourhood of $A$ (continuity property);

$\left(i_{6}\right)$ if $h: X \rightarrow X$ is an odd homeomorphism, $B \subseteq X$ is an open bounded symmetric neighbourhood of 0 and $V$ is a finite dimensional subspace of $X$, then

$$
\gamma(V \cap h(\partial B))=\operatorname{dim} V
$$

$\left(i_{7}\right)$ if $W$ is a closed subspace of $X$ with $\operatorname{codim} W<+\infty$ and $A \in \Sigma$ is such that $\gamma(A)>\operatorname{codim} W$, then $A \cap W \neq \emptyset$.

Let us consider the genus theory $(\Sigma, \mathcal{H}, \gamma)$ on $X$, an even functional $I$ : $X \rightarrow \mathbb{R}$ and $k \in \mathbb{N}$. Then, setting

$$
b_{k}=\inf _{A \in \Sigma_{k}} \sup _{u \in A} I(u)
$$

with

$$
\Sigma_{k}=\{A \in \Sigma: \gamma(A) \geq k\},
$$

the following characterization holds (the proof is essentially contained in [21, Remarks 8.7(i)], nevertheless for completeness here we give more details).

Lemma 2.5. If $\Sigma_{k} \neq \emptyset$ and $b_{k} \in \mathbb{R}$, then

$$
b_{k}=\inf \left\{b \in \mathbb{R}: \gamma\left(I^{b}\right) \geq k\right\} .
$$

Proof. Let us set $\tilde{b}_{k}=\inf \left\{b \in \mathbb{R}: \gamma\left(I^{b}\right) \geq k\right\}$. Taking $b \geq \tilde{b}_{k}$ such that $\gamma\left(I^{b}\right) \geq k$ it results $I^{b} \in \Sigma_{k}$; hence $b_{k} \leq b$ and therefore $\tilde{b}_{k} \geq b_{k}$. Arguing by contradiction, assume $\tilde{b}_{k}>b_{k}$ and take $\bar{b}$ such that $b_{k}<\bar{b}<\tilde{b}_{k}$. Then there exists $\bar{A} \in \Sigma_{k}$ such that $\sup I(u)<\bar{b}$; whence $\bar{A} \subseteq I^{\bar{b}}$ and $\gamma(\bar{A}) \geq k$. By $u \in \bar{A}$

$\left(i_{2}\right)$ it follows $\gamma\left(I^{\bar{b}}\right) \geq k$, hence $\bar{b} \geq \tilde{b}_{k}$, which yields a contradiction.

According to [7], the pseudo-index related to the genus, an even functional $I: X \rightarrow \mathbb{R}$ and $S \in \Sigma$ is the triplet $\left(S, \mathcal{H}^{*}, \gamma^{*}\right)$ such that $\mathcal{H}^{*}$ is a group of odd homeomorphisms and $\gamma^{*}: \Sigma \rightarrow \mathbb{N} \cup\{+\infty\}$ is the map defined by

$$
\gamma^{*}(A)=\min _{h \in \mathcal{H}^{*}} \gamma(h(A) \cap S) \text { for all } A \in \Sigma .
$$

Clearly by $\left(i_{4}\right)$ it follows

$$
\gamma(h(A) \cap S)=\gamma\left(A \cap h^{-1}(S)\right) \quad \text { for all } h \in \mathcal{H}^{*},
$$

then

$$
\gamma^{*}(A)=\min _{h \in \mathcal{H}^{*}} \gamma(A \cap h(S)) \quad \text { for all } \quad A \in \Sigma
$$


The following mini-max theorem was proved in [5, Theorem 2.9] in the setting of Hilbert spaces; the same proof holds on Banach spaces, just taking into account [21, Theorem A.4].

Theorem 2.6. Consider $a, b, c_{0}, c_{\infty} \in \overline{\mathbb{R}},-\infty \leq a<c_{0}<c_{\infty}<b \leq+\infty$. Let $I$ be an even functional, $(\Sigma, \mathcal{H}, \gamma)$ the genus theory on $X, S \in \Sigma,\left(S, \mathcal{H}^{*}, \gamma^{*}\right)$ the pseudo-index theory related to the genus, $I$ and $S$, with

$\mathcal{H}^{*}=\left\{h \in \mathcal{H}: h\right.$ bounded homeomorphism s.t. $h(u)=u$ if $\left.u \notin I^{-1}(] a, b[)\right\}$.

Assume that:

(i) the functional I satisfies $(C P S)$ in $] a, b[$;

(ii) $S \subseteq I^{-1}\left(\left[c_{0},+\infty[)\right.\right.$;

(iii) there exist $\tilde{k} \in \mathbb{N}$ and $\tilde{A} \in \Sigma$ such that $\tilde{A} \subseteq I^{c_{\infty}}$ and $\gamma^{*}(\tilde{A}) \geq \tilde{k}$.

Then the numbers

$$
c_{i}=\inf _{A \in \Sigma_{i}^{*}} \sup _{u \in A} I(u), \quad i \in\{1, \ldots, \tilde{k}\},
$$

with $\Sigma_{i}^{*}=\left\{A \in \Sigma: \gamma^{*}(A) \geq i\right\}$, are critical values for $I$ and

$$
c_{0} \leq c_{1} \leq \cdots \leq c_{\tilde{k}} \leq c_{\infty}
$$

Furthermore, if $c=c_{i}=\cdots=c_{i+r}$, with $i \geq 1$ and $i+r \leq \tilde{k}$, then $\gamma\left(K_{c}\right) \geq$ $r+1$.

In order to apply the theorem above, we need the following result, which allows us to obtain a lower bound for the pseudo-index of a suitable $\tilde{A}$ as in (iii); the proof is alike that of [5, Theorem A.2], nevertheless we prefer to develop here all details, adapting it to Banach spaces.

Theorem 2.7. Let $(\Sigma, \mathcal{H}, \gamma)$ be the genus theory on $X$ and $V, W$ two closed subspaces of $X$. Assume that

$$
\operatorname{dim} V<+\infty \quad \text { and } \quad \operatorname{codim} W<+\infty \text {. }
$$

Then, for every odd bounded homeomorphism $h$ on $X$ and every open bounded symmetric neighbourhood $B$ of 0 in $X$, it results

$$
\gamma(V \cap h(\partial B \cap W)) \geq \operatorname{dim} V-\operatorname{codim} W .
$$

Proof. Taking any odd bounded homeomorphism $h$ on $X$ and any open bounded symmetric neighbourhood $B$ of 0 in $X$, as the set $V \cap h(\partial B \cap W)$ is compact, by property $\left(i_{5}\right)$ there exists $\delta>0$ such that

$$
\gamma\left(N_{\delta}(V \cap h(\partial B \cap W))\right)=\gamma(V \cap h(\partial B \cap W)),
$$

where $N_{\delta}(V \cap h(\partial B \cap W))$ is the closed $\delta$-neighbourhood of $V \cap h(\partial B \cap W)$.

We claim that there exists $\varepsilon>0$ such that, setting $W_{\varepsilon}=N_{\varepsilon}(W)$, it results

$$
V \cap h\left(\partial B \cap W_{\varepsilon}\right) \subseteq N_{\delta}(V \cap h(\partial B \cap W))
$$


Indeed, arguing by contradiction, let us assume that for any $n \in \mathbb{N}$ there exists $y_{n} \in V \cap h\left(\partial B \cap W_{\frac{1}{n}}\right)$ which does not belong to $N_{\delta}(V \cap h(\partial B \cap W))$, or equivalently,

$\forall n \in \mathbb{N} \exists x_{n} \in \partial B \cap W_{\frac{1}{n}}$ s.t. $h\left(x_{n}\right)=y_{n} \in V \quad$ and $\quad d\left(y_{n}, V \cap h(\partial B \cap W)\right)>\delta$.

Being $\left(y_{n}\right)_{n}$ bounded in the finite dimensional subspace $V$, it converges, up to subsequences, to a certain $\bar{y} \in V$ and

$$
d(\bar{y}, V \cap h(\partial B \cap W)) \geq \delta .
$$

Then, denoting $h^{-1}(\bar{y})$ by $\bar{x}$, as $h$ is an homeomorphism, it follows that $\left(x_{n}\right)_{n}$ converges to $\bar{x}$. So, $\bar{x} \in \partial B \cap W$ is such that $\bar{y}=h(\bar{x}) \in h(\partial B \cap W) \cap V$ while (2.13) holds, which yields a contradiction and (2.12) is satisfied.

By (2.11), (2.12) and property $\left(i_{2}\right)$ it follows that

$$
\gamma\left(V \cap h\left(\partial B \cap W_{\varepsilon}\right)\right)=\gamma(V \cap h(\partial B \cap W)) .
$$

Now, let us set

$$
R_{\varepsilon}=\overline{X \backslash W_{\varepsilon}}
$$

By definition, $R_{\varepsilon} \cap W=\emptyset$. As codim $W<+\infty$, by property $\left(i_{7}\right)$ it must be

$$
\gamma\left(R_{\varepsilon}\right) \leq \operatorname{codim} W
$$

Moreover, by the definition of the subset $R_{\varepsilon}$, it is

$$
h(\partial B)=h\left(\partial B \cap W_{\varepsilon}\right) \cup h\left(\partial B \cap R_{\varepsilon}\right),
$$

hence by property $\left(i_{3}\right)$ and $(2.14)$ it results

$$
\gamma(V \cap h(\partial B)) \leq \gamma(V \cap h(\partial B \cap W))+\gamma\left(V \cap h\left(\partial B \cap R_{\varepsilon}\right)\right) .
$$

Furthermore by $\left(i_{6}\right)$ it is

$$
\gamma(V \cap h(\partial B))=\operatorname{dim} V
$$

on the other hand, by $\left(i_{2}\right),\left(i_{4}\right)$ and $(2.15)$ it follows

$$
\gamma\left(V \cap h\left(\partial B \cap R_{\varepsilon}\right)\right) \leq \gamma\left(h\left(\partial B \cap R_{\varepsilon}\right)\right) \leq \gamma\left(\partial B \cap R_{\varepsilon}\right) \leq \gamma\left(R_{\varepsilon}\right) \leq \operatorname{codim} W .
$$

Finally, by (2.16)-(2.18), we can conclude that

$$
\operatorname{dim} V \leq \gamma(V \cap h(\partial B \cap W))+\operatorname{codim} W
$$

and the proof is complete.

Remark 2.8. Under the assumptions of Theorem 2.7, for every odd bounded homeomorphism $h$ on $X$ and every open bounded symmetric neighbourhood $B$ of 0 in $X$, we have also

$$
\gamma(W \cap h(\partial B \cap V)) \geq \operatorname{dim} V-\operatorname{codim} W .
$$

Indeed, since $h(\partial B)=\partial(h(B))$ (cf. [7, p. 414]) is the boundary of the open bounded symmetric neighbourhood $h(B)$ of 0 , by property $\left(i_{4}\right)$ it results

$$
\gamma(W \cap h(\partial B \cap V))=\gamma\left(h^{-1}(W) \cap(\partial B \cap V)\right)=\gamma\left(h^{-1}(W \cap h(\partial B)) \cap V\right),
$$

hence the proof follows by Theorem 2.7. 
Starting from the genus we can define the sequence of eigenvalues $\left(\mu_{k}\right)_{k}$ as in [18]:

$$
\mu_{k}=\inf _{A \in \Sigma_{k}} \sup _{u \in A \backslash\{0\}} \frac{\int_{\Omega}|\nabla u|^{p} \mathrm{~d} x}{\int_{\Omega}|u|^{p} \mathrm{~d} x},
$$

where $\Sigma_{k}$ is as in $(2.8)$ with $X=W_{0}^{1, p}(\Omega)$. Using the properties of the genus recalled above, we obtain a comparison among $\left(\mu_{k}\right)_{k}$ and the sequences of quasi-eigenvalues $\left(\eta_{k}\right)_{k},\left(\nu_{k}\right)_{k}$.

Proposition 2.9. For all $k \in \mathbb{N}$ it results $\eta_{k} \leq \mu_{k} \leq \nu_{k}$.

Proof. As we have already pointed out, the second inequality follows by [16, Remark 1.1(4)]. So in order to prove the first one, by contradiction let us assume that there exists $k \in \mathbb{N}$ such that $\mu_{k}<\eta_{k}$. Fix $\varepsilon>0$ such that $\mu_{k}+\varepsilon<\eta_{k}$.

Let us consider the even $C^{1}$ functional

$$
\Psi(u)=\int_{\Omega}|\nabla u|^{p} \mathrm{~d} x \quad \text { on } \quad W_{0}^{1, p}(\Omega)
$$

and the manifold $\mathcal{S}_{p}=\left\{u \in W_{0}^{1, p}(\Omega):|u|_{p}=1\right\}$. Since by $[10$, Lemmas 5.1 and 5.3] it results

$$
\eta_{k}=\min _{u \in W_{k-1} \cap \mathcal{S}_{p}} \Psi(u)
$$

with $W_{k-1}$ such that (2.3)-(2.4) hold for $h=k-1$, it follows

$$
W_{k-1} \cap \mathcal{S}_{p} \subseteq\left(\left.\Psi\right|_{\mathcal{S}_{p}}\right)_{\eta_{k}}
$$

thus we get

$$
\left\{u \in \mathcal{S}_{p}: \Psi(u)<\eta_{k}\right\} \subseteq \mathcal{S}_{p} \backslash W_{k-1} .
$$

As by $[12$, Lemma 4$]$ the functional $\left.\Psi\right|_{\mathcal{S}_{p}}$ satisfies the Palais-Smale condition on ]0, $+\infty[$, by Lemma 2.5 the following characterization holds:

$$
\mu_{k}=\inf \left\{c \in \mathbb{R}: \gamma\left(\left(\left.\Psi\right|_{\mathcal{S}_{p}}\right)^{c}\right) \geq k\right\} \quad \text { for all } k \in \mathbb{N} .
$$

Then there exists $\bar{c} \in \mathbb{R}$ such that

$$
\mu_{k} \leq \bar{c}<\mu_{k}+\varepsilon \quad \text { and } \quad \gamma\left(\left(\left.\Psi\right|_{\mathcal{S}_{p}}\right)^{\bar{c}}\right) \geq k .
$$

On the other hand,

$$
\left(\left.\Psi\right|_{\mathcal{S}_{p}}\right)^{\bar{c}} \subseteq\left(\left.\Psi\right|_{\mathcal{S}_{p}}\right)^{\mu_{k}+\varepsilon} \subseteq\left\{u \in \mathcal{S}_{p}: \Psi(u)<\eta_{k}\right\}
$$

hence by $(2.19)$ it results $\left(\left.\Psi\right|_{\mathcal{S}_{p}}\right)^{\bar{c}} \cap W_{k-1}=\emptyset$ and, by property $\left(i_{7}\right)$ and $(2.3)$, it must be

$$
\gamma\left(\left(\left.\Psi\right|_{\mathcal{S}_{p}}\right)^{\bar{c}}\right) \leq k-1,
$$

in contradiction with (2.20). 


\section{The multiplicity results}

From $\left(H_{0}\right)$ and $\left(H_{1}\right)$ we have that for all $\sigma>0$ there exists $A_{\sigma}>0$ such that

$$
|f(x, t)| \leq \sigma|t|^{p-1}+A_{\sigma} \quad \text { for a.e. } x \in \Omega \text { and for all } t \in \mathbb{R} .
$$

Thus, the functional

$$
J(u)=\frac{1}{p} \int_{\Omega}|\nabla u|^{p} \mathrm{~d} x-\frac{l_{\infty}}{p} \int_{\Omega}|u|^{p} \mathrm{~d} x-\int_{\Omega} F(x, u) \mathrm{d} x \quad \text { on } W_{0}^{1, p}(\Omega),
$$

with $F(x, t)=\int_{0}^{t} f(x, s) \mathrm{d} s$, is $C^{1}$ (cf. e.g. [11, Theorem 9 and p. 355]) and the weak solutions of problem $\left(P_{\infty}\right)$ are its critical points.

In the next proposition we prove that the functional $J$ satisfies the $(C P S)$ condition both in the non-resonant case (i.e. under assumption $\left(H_{3}\right)$ ) and in the resonant one (but assuming also $\left(H_{6}\right)$ ). We point out that this property is proved in [16, Proposition 3.1] by using the continuity of $f$ on $\bar{\Omega} \times \mathbb{R}$ instead of $\left(H_{0}\right)$ and, at least in the non-resonant case, also hypotheses $\left(H_{2}\right)$ and $\left(H_{5}\right)$; here, we remove such assumptions.

Proposition 3.1. Assume that $\left(H_{0}\right)-\left(H_{1}\right)$ hold. Then

(i) if $\left(\mathrm{H}_{3}\right)$ holds, the functional $J$ in (3.2) satisfies $(C P S)$ in $\mathbb{R}$;

(ii) if $\left(H_{3}^{\prime}\right)$ and $\left(H_{6}\right)$ hold, the functional $J$ in (3.2) satisfies $(C P S)$ in $\mathbb{R}$.

Proof. (i) Let $c \in \mathbb{R}$ and $\left(u_{n}\right)_{n}$ be a sequence in $W_{0}^{1, p}(\Omega)$ such that $(2.7)$ holds, so

$$
\begin{aligned}
& \frac{1}{p} \int_{\Omega}\left|\nabla u_{n}\right|^{p} \mathrm{~d} x-\frac{l_{\infty}}{p} \int_{\Omega}\left|u_{n}\right|^{p} \mathrm{~d} x-\int_{\Omega} F\left(x, u_{n}\right) \mathrm{d} x=c+o(1), \\
& \int_{\Omega}\left|\nabla u_{n}\right|^{p} \mathrm{~d} x-l_{\infty} \int_{\Omega}\left|u_{n}\right|^{p} \mathrm{~d} x-\int_{\Omega} f\left(x, u_{n}\right) u_{n} \mathrm{~d} x=o(1) \\
& \int_{\Omega}\left|\nabla u_{n}\right|^{p-2} \nabla u_{n} \cdot \nabla \varphi \mathrm{d} x-l_{\infty} \int_{\Omega}\left|u_{n}\right|^{p-2} u_{n} \varphi \mathrm{d} x \\
& \quad-\int_{\Omega} f\left(x, u_{n}\right) \varphi \mathrm{d} x=o(1),
\end{aligned}
$$

for all $\varphi \in W_{0}^{1, p}(\Omega)$, where $o(1)$ denotes an infinitesimal sequence.

In order to prove the statement, it is enough to show that $\left(\left\|u_{n}\right\|\right)_{n}$ is bounded (see e.g. [11, Lemma 2]). Then, arguing by contradiction, assume that

$$
\left\|u_{n}\right\| \rightarrow+\infty \quad \text { as } n \rightarrow+\infty .
$$

Hence, without loss of generality, we can assume that $\left\|u_{n}\right\|>0$ for all $n \in \mathbb{N}$ and set $w_{n}=\frac{u_{n}}{\left\|u_{n}\right\|}$; of course $\left(w_{n}\right)_{n}$ is bounded in $W_{0}^{1, p}(\Omega)$ and there exists $w \in W_{0}^{1, p}(\Omega)$ such that, up to subsequences, it results

$$
\begin{array}{ll}
w_{n} \rightarrow w & \text { weakly in } W_{0}^{1, p}(\Omega), \\
w_{n} \rightarrow w & \text { strongly in } L^{s}(\Omega), \quad \text { for all } \quad s \in\left[p, p^{*}[.\right.
\end{array}
$$


Dividing (3.3) and (3.4) by $\left\|u_{n}\right\|^{p}$ and (3.5) by $\left\|u_{n}\right\|^{p-1}$ respectively, we get

$$
\begin{aligned}
& 1=\int_{\Omega}\left|\nabla w_{n}\right|^{p} \mathrm{~d} x=l_{\infty}\left|w_{n}\right|_{p}^{p}+p \int_{\Omega} \frac{F\left(x, u_{n}\right)}{\left\|u_{n}\right\|^{p}} \mathrm{~d} x+o(1), \\
& 1=\int_{\Omega}\left|\nabla w_{n}\right|^{p} \mathrm{~d} x=l_{\infty}\left|w_{n}\right|_{p}^{p}+\int_{\Omega} \frac{f\left(x, u_{n}\right) u_{n}}{\left\|u_{n}\right\|^{p}} \mathrm{~d} x+o(1), \\
& \int_{\Omega}\left|\nabla w_{n}\right|^{p-2} \nabla w_{n} \cdot \nabla \varphi \mathrm{d} x=l_{\infty} \int_{\Omega}\left|w_{n}\right|^{p-2} w_{n} \varphi \mathrm{d} x \\
& \quad+\int_{\Omega} \frac{f\left(x, u_{n}\right)}{\left\|u_{n}\right\|^{p-1}} \varphi \mathrm{d} x+o(1),
\end{aligned}
$$

for all $\varphi \in W_{0}^{1, p}(\Omega)$.

Next, we claim that $w \neq 0$. In fact, if $w=0$, by (3.8) and (3.9) it follows

$$
1=\int_{\Omega} \frac{f\left(x, u_{n}\right) u_{n}}{\left\|u_{n}\right\|^{p}} \mathrm{~d} x+o(1)
$$

on the other hand, by (3.1) we have

$$
\left|\int_{\Omega} \frac{f\left(x, u_{n}\right) u_{n}}{\left\|u_{n}\right\|^{p}} \mathrm{~d} x\right| \leq \sigma\left|w_{n}\right|_{p}^{p}+\frac{A_{\sigma}}{\left\|u_{n}\right\|^{p-1}}\left|w_{n}\right|_{1}
$$

thus (3.6) and again (3.8) imply

$$
\lim _{n \rightarrow+\infty} \int_{\Omega} \frac{f\left(x, u_{n}\right) u_{n}}{\left\|u_{n}\right\|^{p}} \mathrm{~d} x=0
$$

in contradiction with (3.11).

Now, subtracting by (3.9) equation (3.10) evaluated in $\varphi=w$, it follows

$$
\begin{aligned}
& \int_{\Omega}\left|\nabla w_{n}\right|^{p-2} \nabla w_{n} \cdot\left(\nabla w_{n}-\nabla w\right) \mathrm{d} x \\
& =l_{\infty} \int_{\Omega}\left|w_{n}\right|^{p-2} w_{n}\left(w_{n}-w\right) \mathrm{d} x \\
& \quad+\int_{\Omega} \frac{f\left(x, u_{n}\right)}{\left\|u_{n}\right\|^{p-1}}\left(w_{n}-w\right) \mathrm{d} x+o(1) .
\end{aligned}
$$

We observe that (3.8) implies

$$
\left.\left.\left|\int_{\Omega}\right| w_{n}\right|^{p-2} w_{n}\left(w_{n}-w\right) \mathrm{d} x|\leq| w_{n}\right|_{p} ^{p-1}\left|w_{n}-w\right|_{p}=o(1)
$$

while by (3.1), (3.6) and (3.8) it follows

$$
\left|\int_{\Omega} \frac{f\left(x, u_{n}\right)}{\left\|u_{n}\right\|^{p-1}}\left(w_{n}-w\right) \mathrm{d} x\right| \leq \sigma\left|w_{n}\right|_{p}^{p-1}\left|w_{n}-w\right|_{p}+\frac{A_{\sigma}}{\left\|u_{n}\right\|^{p-1}}\left|w_{n}-w\right|_{1}=o(1) .
$$

Hence, by (3.12)

$$
\int_{\Omega}\left|\nabla w_{n}\right|^{p-2} \nabla w_{n} \cdot\left(\nabla w_{n}-\nabla w\right) \mathrm{d} x=o(1)
$$

thus, by [11, Theorem 10] it follows

$$
w_{n} \rightarrow w \quad \text { strongly in } \quad W_{0}^{1, p}(\Omega) .
$$


Finally, by using (3.1), (3.6) and (3.8), it follows

$$
\lim _{n \rightarrow+\infty} \int_{\Omega} \frac{f\left(x, u_{n}\right)}{\left\|u_{n}\right\|^{p-1}} \varphi \mathrm{d} x=0 \quad \text { for all } \varphi \in W_{0}^{1, p}(\Omega) .
$$

So, by (3.13) and (3.14), passing to the limit in (3.10), we get

$$
\int_{\Omega}|\nabla w|^{p-2} \nabla w \cdot \nabla \varphi \mathrm{d} x=l_{\infty} \int_{\Omega}|w|^{p-2} w \varphi \mathrm{d} x \quad \text { for all } \quad \varphi \in W_{0}^{1, p}(\Omega) ;
$$

this means that $l_{\infty}$ is an eigenvalue of $-\Delta_{p}$ in $W_{0}^{1, p}(\Omega)$, against assumption $\left(H_{3}\right)$, thus the proof is complete.

(ii) The proof is word by word as in [16, Proposition 3.1(ii)].

Proof of Theorem 1.1. By Proposition 3.1(i) the functional $J$ in (3.2) satisfies $(C P S)$ in $\mathbb{R}$ and by assumption $\left(H_{5}\right)$ it is even. Moreover, $\left(H_{4}\right)$ implies $l_{0} \neq 0$. In order to have a control on $F$ near to $t=0$, let us point out that from $\left(H_{1}\right)$ and $\left(\mathrm{H}_{2}\right)$ it follows that there exist

$$
\lim _{|t| \rightarrow+\infty} \frac{F(x, t)}{|t|^{p}}=0 \quad \text { uniformly with respect to a.e. } x \in \Omega
$$

and

$$
\lim _{t \rightarrow 0} \frac{F(x, t)}{|t|^{p}}=\frac{l_{0}}{p} \quad \text { uniformly with respect to a.e. } x \in \Omega ;
$$

hence for any $\sigma>0$ there exist $R_{\sigma}, \delta_{\sigma}>0$ (without loss of generality $R_{\sigma} \geq 1$ ) such that

$$
\begin{aligned}
& |F(x, t)| \leq \frac{\sigma}{p}|t|^{p} \quad \text { if }|t|>R_{\sigma} \text {, for a.e. } x \in \Omega, \\
& \left.\left.\left|F(x, t)-\frac{l_{0}}{p}\right| t\right|^{p}\left|\leq \frac{\sigma}{p}\right| t\right|^{p} \quad \text { if }|t|<\delta_{\sigma} \text {, for a.e. } x \in \Omega .
\end{aligned}
$$

Moreover, by $\left(H_{0}\right)$, taking any $s \in\left[0, p^{*}-p\left[\left(p^{*}-p=+\infty\right.\right.\right.$ if $\left.p \geq N\right)$, there exists $a_{R_{\sigma}}>0$ such that, if $\delta_{\sigma} \leq|t| \leq R_{\sigma}$ and for a.e. $x \in \Omega$, we have

$$
|F(x, t)| \leq a_{R_{\sigma}}|t|^{s+p} .
$$

At first we restrict to the case $l_{0}<0$, so that $\left(H_{4}\right)$ becomes

$$
l_{0}+l_{\infty}<\eta_{h} \leq \nu_{k}<l_{\infty} .
$$

Therefore, (3.15)-(3.17) imply that for any $\sigma>0$ there exists $a_{\sigma}>0$ large enough such that for a.e. $x \in \Omega$ and for all $t \in \mathbb{R}$

$$
-\frac{\left(\sigma-l_{0}\right)}{p}|t|^{p}-a_{\sigma}|t|^{s+p} \leq F(x, t) \leq \frac{\left(\sigma+l_{0}\right)}{p}|t|^{p}+a_{\sigma}|t|^{s+p},
$$

which in particular implies

$$
\int_{\Omega} F(x, u) \mathrm{d} x \leq \frac{\left(\sigma+l_{0}\right)}{p}|u|_{p}^{p}+a_{\sigma}|u|_{s+p}^{s+p} \quad \text { for all } \quad u \in W_{0}^{1, p}(\Omega) .
$$

By the Sobolev inequalities for all $u \in W_{0}^{1, p}(\Omega)$ it results

$$
J(u) \geq \frac{1}{p}\|u\|^{p}-\frac{l_{\infty}+l_{0}+\sigma}{p}|u|_{p}^{p}-a_{\sigma}^{\prime}\|u\|^{s+p}
$$


for a suitable $a_{\sigma}^{\prime}>0$. Now let us consider $\eta_{h}$ as in (3.18); by (2.3) it is $W_{0}^{1, p}(\Omega)=V_{h-1} \oplus W_{h-1}$, where $V_{h-1}=\operatorname{span}\left\{\psi_{1}, \ldots, \psi_{h-1}\right\}$ and $W_{h-1}$ is its complement.

Thus by (3.19) and (2.4) for all $u \in W_{h-1}$ it results

$$
J(u) \geq \frac{1}{p}\left(1-\frac{l_{\infty}+l_{0}+\sigma}{\eta_{h}}\right)\|u\|^{p}-a_{\sigma}^{\prime}\|u\|^{s+p}
$$

hence, by (3.18) and for $\sigma$ small enough, there exists $a_{\sigma}^{\prime \prime}>0$ such that

$$
J(u) \geq a_{\sigma}^{\prime \prime}\|u\|^{p}-a_{\sigma}^{\prime}\|u\|^{s+p} \quad \text { for all } \quad u \in W_{h-1} .
$$

So, if $\rho$ is small enough there exists $c_{0}>0$ such that

$$
J(u) \geq c_{0} \quad \text { for all } \quad u \in S_{\rho} \cap W_{h-1} .
$$

On the other hand we notice that by (3.1), (3.2) and the Cauchy-Schwarz inequality, fixing any $\sigma>0, B_{\sigma}>0$ exists such that

$$
J(u) \leq \frac{1}{p}\|u\|^{p}-\frac{l_{\infty}}{p}|u|_{p}^{p}+\frac{\sigma}{2 p}|u|_{p}^{p}+B_{\sigma}|u|_{p} \quad \text { for all } \quad u \in W_{0}^{1, p}(\Omega) .
$$

Let us consider $\nu_{k}$ as in (3.18) and take $\sigma>0$ such that $\nu_{k}+\sigma<l_{\infty}$. From definition (2.6), for such a fixed $\sigma>0$ there exists a subspace $V_{k}^{\sigma}$ in $\mathbb{W}_{k}$ (see (2.5)), with $\operatorname{dim} V_{k}^{\sigma} \geq k$, such that

$$
\nu_{k} \leq \sup _{u \in V_{k}^{\sigma} \backslash\{0\}} \frac{\|u\|^{p}}{|u|_{p}^{p}}<\nu_{k}+\frac{\sigma}{2} .
$$

Hence,

$$
J(u) \leq \frac{1}{p}\left(\nu_{k}+\sigma-l_{\infty}\right)|u|_{p}^{p}+B_{\sigma}|u|_{p} \quad \text { for all } \quad u \in V_{k}^{\sigma}
$$

and, as without loss of generality we can assume that $V_{k}^{\sigma}$ is a $k$-dimensional subspace, the functional $J$ tends to $-\infty$ as $\|u\|$ diverges in $V_{k}^{\sigma}$, so there exists $c_{\infty}>c_{0}$ such that

$$
J(u) \leq c_{\infty} \quad \text { for all } u \in V_{k}^{\sigma} .
$$

Now, considering the pseudo-index theory $\left(S_{\rho} \cap W_{h-1}, \mathcal{H}^{*}, \gamma^{*}\right)$ related to the genus, $S_{\rho} \cap W_{h-1}$ and $J$, by Theorem 2.7 (applied to $V=V_{k}^{\sigma}, \partial B=S_{\rho}$ and $\left.W=W_{h-1}\right)$ we get

$\gamma\left(V_{k}^{\sigma} \cap h\left(S_{\rho} \cap W_{h-1}\right)\right) \geq \operatorname{dim} V_{k}^{\sigma}-\operatorname{codim} W_{h-1} \quad$ for all $h \in \mathcal{H}^{*}$, hence (2.9) implies

$$
\gamma^{*}\left(V_{k}^{\sigma}\right) \geq k-h+1
$$

So, Theorem 2.6 applies with $\tilde{A}:=V_{k}^{\sigma}$ and $S:=S_{\rho} \cap W_{h-1}$ and $J$ has at least $k-h+1$ distinct pairs of critical points corresponding to at most $k-h+1$ distinct critical values $c_{i}$, where $c_{i}$ is as in (2.10).

Next, let us consider the case $l_{0}>0$, so that $\left(H_{4}\right)$ becomes

$$
l_{\infty}<\eta_{h} \leq \nu_{k}<l_{0}+l_{\infty} .
$$

Then, fixing $\sigma>0$ such that

$$
l_{\infty}+\sigma<\eta_{h} \leq \nu_{k}+\sigma<l_{0}+l_{\infty},
$$


arguments similar to those ones used before imply that

$$
J(u) \geq \frac{1}{p}\left(1-\frac{l_{\infty}+\sigma}{\eta_{h}}\right)\|u\|^{p}-B_{\sigma}\|u\| \quad \text { for all } \quad u \in W_{h-1}
$$

and

$$
J(u) \leq-\frac{1}{p}\left(l_{\infty}+l_{0}-\nu_{k}-\sigma\right)|u|_{p}^{p}+a_{\sigma}|u|_{s+p}^{s+p} \quad \text { for all } \quad u \in V_{k}^{\sigma},
$$

for suitable positive constants $B_{\sigma}$ and $a_{\sigma}$. Thus, Theorem 2.6 applies to the functional $-J$, with $\tilde{A}:=W_{h-1}$ and $S:=S_{\rho} \cap V_{k}^{\sigma}$, by using Remark 2.8.

Proof of Theorem 1.2. By Proposition 3.1(ii) the functional $J$ satisfies (CPS) and we can proceed as in the proof of Theorem 1.1.

Remark 3.2. If $\left(H_{2}\right)$ and $\left(H_{4}\right)$ are replaced by $\left(H_{2}^{\prime}\right)$ there exists

$$
\lim _{t \rightarrow 0} \frac{f(x, t)}{|t|^{p-2} t}=-\infty \quad \text { uniformly with respect to a.e. } x \in \Omega ;
$$

$\left(H_{4}^{\prime}\right) \nu_{k}<l_{\infty}$ for some $k \in \mathbb{N}$,

then by Proposition $3.1\left(P_{\infty}\right)$ has at least $k$ distinct pairs of non-trivial solutions, choosing in Theorem 2.6 $\tilde{A}=V_{k}^{\sigma}$ and $S=S_{\rho}$.

On the other hand, if $\left(H_{2}\right)$ and $\left(H_{4}\right)$ are replaced by $\left(H_{2}^{\prime \prime}\right)$ there exists

$$
\lim _{t \rightarrow 0} \frac{f(x, t)}{|t|^{p-2} t}=+\infty \quad \text { uniformly with respect to a.e. } x \in \Omega,
$$

then $\left(P_{\infty}\right)$ has infinitely many pairs of non-trivial solutions. Indeed, by Proposition 3.1 , fixing $h$ such that $\eta_{h}>l_{\infty}$, for any $k>h$ we can apply Theorem 2.6 to the functional $-J$ with $\tilde{A}=W_{h-1}$ and $S=S_{\rho} \cap \tilde{V}_{k}^{\sigma}$, where $\tilde{V}_{k}^{\sigma}$ is any $k$-dimensional subspace of $W_{0}^{1, p}(\Omega)$, thus obtaining $k-h+1$ pairs of solutions. The conclusion follows by the arbitrariness of $k$.

Remark 3.3. The arguments in Remark 3.2 still work in the resonant case.

\section{The existence results}

Proof of Theorem 1.4. By Proposition 3.1(i) the functional $J$ in (3.2) satisfies $(C P S)$ in $\mathbb{R}$. At first we restrict to the case $l_{0}<0$, so that $\left(H_{7}\right)$ becomes

$$
\nu_{k-1}<l_{0}+l_{\infty}<\eta_{k} \leq \nu_{k}<l_{\infty}
$$

Let us fix $\sigma \in] 0, \varepsilon_{0}\left[\right.$, with $\varepsilon_{0}$ as in $\left(H_{8}\right)$, such that $\nu_{k}+2 \sigma<l_{\infty}$ and $l_{0}+l_{\infty}+\sigma<$ $\eta_{k}$. From (2.6) there exists a subspace $V_{k-1}^{\sigma}$ in $\mathbb{W}_{k-1}$ (see $\left.(2.5)\right)$, which can be chosen with $\operatorname{dim} V_{k-1}^{\sigma}=k-1$, such that

$$
\nu_{k-1} \leq \sup _{u \in V_{k-1}^{\sigma} \backslash\{0\}} \frac{\|u\|^{p}}{|u|_{p}^{p}}<\nu_{k-1}+\sigma .
$$


Hence, recalling that $\left(\nu_{k}\right)_{k}$ is an increasing sequence, so in particular $\nu_{k-1}+\sigma<$ $l_{\infty}$, by $(4.2)$ and $\left(H_{8}\right)$ for all $u \in V_{k-1}^{\sigma}$ it results

$$
J(u) \leq \frac{1}{p}\left(\nu_{k-1}+\sigma-l_{\infty}\right)|u|_{p}^{p}-\int_{\Omega} F(x, u) \mathrm{d} x \leq 0
$$

On the other hand, since by $(2.4)$

$$
\eta_{k} \int_{\Omega}|u|^{p} \mathrm{~d} x \leq \int_{\Omega}|\nabla u|^{p} \mathrm{~d} x \quad \text { for all } u \in W_{k-1},
$$

proceeding as in the proof of Theorem 1.1, by using (3.20) (with $h=k$ ) and (4.1), for a suitable $\rho$ we get the existence of $\beta>0$ such that

$$
J(u) \geq \beta \text { for all } u \in S_{\rho} \cap W_{k-1} .
$$

We claim that

$$
W_{0}^{1, p}(\Omega)=V_{k-1}^{\sigma} \oplus W_{k-1},
$$

which means that under our assumptions $V_{k-1}=\operatorname{span}\left\{\psi_{1}, \ldots, \psi_{k-1}\right\}$ in $(2.3)$ is indeed $V_{k-1}^{\sigma}$. To this aim we prove that $V_{k-1}^{\sigma} \cap W_{k-1}=\{0\}$ : otherwise, if there exists $\bar{u} \in\left(V_{k-1}^{\sigma} \cap W_{k-1}\right) \backslash\{0\}$, choosen $\rho$ as in $(4.5), \rho \frac{\bar{u}}{\|\bar{u}\|} \in V_{k-1}^{\sigma} \cap$ $\left(S_{\rho} \cap W_{k-1}\right)$, thus (4.3) and (4.5) yield a contradiction.

Now, again, from definition (2.6) there exists a subspace $V^{\sigma}$ in $\mathbb{W}_{k}$, which can be chosen with $\operatorname{dim} V^{\sigma} \geq \bar{k}$, being $\bar{k}$ as in (1.3), such that

$$
\nu_{k} \leq \sup _{u \in V^{\sigma} \backslash\{0\}} \frac{\|u\|^{p}}{|u|_{p}^{p}}<\nu_{k}+\sigma .
$$

Next we show that $\operatorname{dim} V^{\sigma}=\bar{k}$ and

$$
V^{\sigma}=V_{k-1}^{\sigma} \oplus \operatorname{span}\left\{\psi_{k}, \ldots, \psi_{\bar{k}}\right\}=\operatorname{span}\left\{\psi_{1}, \ldots, \psi_{\bar{k}}\right\},
$$

where by [10, Lemma 5.1], $\left(\psi_{i}\right)_{i} \subset W_{0}^{1, p}(\Omega)$ is a sequence such that for all $i \in \mathbb{N}:\left|\psi_{i}\right|_{p}=1$ and $\eta_{i}=\left\|\psi_{i}\right\|^{p}$. Let us consider the quasi-eigenvalue $\eta_{\bar{k}+1}$, thus, from (1.3) we can also choose $\sigma$ so that $\nu_{k}+\sigma<\eta_{\bar{k}+1}$. Assume by contradiction that $\psi_{j}$ belongs to $V^{\sigma}$ for some $j \geq \bar{k}+1$; then by (4.7) and the monotonicity of the sequence $\left(\eta_{k}\right)_{k}$ (see Sect. 2) it follows

$$
\eta_{j} \geq \eta_{\bar{k}+1}>\nu_{k}+\sigma>\sup _{u \in V^{\sigma} \backslash\{0\}} \frac{\|u\|^{p}}{|u|_{p}^{p}} \geq\left\|\psi_{j}\right\|^{p}=\eta_{j},
$$

which is a contradiction, so (4.8) is proved.

By (3.1) it results

$$
J(u) \leq \frac{1}{p}\left(\nu_{k}+2 \sigma-l_{\infty}\right)|u|_{p}^{p}+B_{\sigma}|u|_{p} \quad \text { for all } \quad u \in V^{\sigma} ;
$$

thus, by our choice of $\sigma$ the functional $J$ tends to $-\infty$ as $\|u\|$ diverges in $V^{\sigma}$ and both there exists $R_{2}>0$ such that

$$
J(u) \leq 0 \quad \text { if } u \in V^{\sigma},\|u\| \geq R_{2}
$$

and there exists $\gamma \in \mathbb{R}$ such that

$$
J(u) \leq \gamma \quad \text { for all } u \in V^{\sigma} .
$$


Let us remark that by Example 2.3, (4.6) and (4.8), setting $V:=V_{k-1}^{\sigma}, S:=$ $S_{\rho} \cap W_{k-1}, e:=\frac{\psi_{k}}{\left\|\psi_{k}\right\|}$, it results that $S$ and $\partial Q=V_{k-1}^{\sigma} \cap S_{R_{1}}$ link for $R_{1}>\rho$. Then by (4.3), (4.5) and (4.9), Theorem 2.4 applies for $R_{1}>R_{2}$, so there exists a critical level $c \geq \beta>0$ corresponding to a non-trivial solution of $\left(P_{\infty}\right)$.

Now, let us consider the case $l_{0}>0$, so that $\left(H_{7}\right)$ becomes

$$
l_{\infty}<\eta_{k} \leq \nu_{k}<l_{0}+l_{\infty} .
$$

As $\nu_{k-1}<l_{\infty}\left(\right.$ see $\left(H_{8}\right)$ and Remark 1.7), let us fix $\left.\sigma \in\right] 0, \varepsilon_{0}[$ such that $\nu_{k-1}+\sigma<l_{\infty}, l_{\infty}+\sigma<\eta_{k}, \nu_{k}+2 \sigma<l_{0}+l_{\infty}$ and consider $V_{k-1}^{\sigma}$ in $\mathbb{W}_{k-1}$ such that $\operatorname{dim} V_{k-1}^{\sigma}=k-1$ and (4.2) holds.

By (3.1), (4.4) and the choice of $\sigma$, there exists $\beta \in \mathbb{R}$ such that

$$
J(u) \geq \beta \text { for all } u \in W_{k-1} .
$$

Furthermore, by $\left(H_{8}\right)$ and the choice of $\sigma$ there exists $\delta_{1}>0$ such that

$$
J(u) \leq \frac{\left(\sigma-\varepsilon_{0}\right)}{p}|u|_{p}^{p} \leq-\delta_{1}\|u\|^{p} \quad \text { for all } \quad u \in V_{k-1}^{\sigma},
$$

then not only

$$
\sup _{u \in V_{k-1}^{\sigma}} J(u)<+\infty
$$

but also there exist $R>0$ large enough and $\alpha<\beta$ such that

$$
J(u) \leq \alpha \quad \text { for all } \quad u \in V_{k-1}^{\sigma} \cap S_{R} .
$$

Hence, setting $Q=V_{k-1}^{\sigma} \cap B_{R}$ and $S=W_{k-1}$, from $\alpha<\beta$ it follows (4.6), then by Example 2.2 and Theorem 2.4 there exists a critical level $c$ of $J$ such that

$$
\beta \leq c \leq \sup _{u \in Q} J(u)=0 .
$$

Next we show that $c<0$, so $\left(P_{\infty}\right)$ admits a non-trivial solution. To this aim we prove that there exists a function $\bar{\phi} \in C\left(W_{0}^{1, p}(\Omega), W_{0}^{1, p}(\Omega)\right)$, with $\left.\bar{\phi}\right|_{\partial Q}=\mathrm{id}$, such that

$$
\sup _{u \in Q} J(\bar{\phi}(u))<0 .
$$

At first we observe that, reasoning as before, by (1.3) there exists $V^{\sigma}$ in $\mathbb{W}_{k}$, such that $\operatorname{dim} V^{\sigma}=\bar{k}$ and (4.7)-(4.8) hold; so, without loss of generality, (2.2) is verified by a suitable $L>1$.

Then, by (3.21) and the choice of $\sigma$, there exist $\rho \in] 0, R\left[\right.$ and $\delta_{2}>0$ such that

$$
J(u) \leq-\delta_{2} \quad \text { for all } u \in V^{\sigma} \quad \text { with } \quad \frac{\rho}{L} \leq\|u\| \leq \sqrt{2} \rho .
$$

Now, we can define $\bar{\phi}: V_{k-1}^{\sigma} \rightarrow W_{0}^{1, p}(\Omega)$ (which can be continuously extended to $\left.W_{0}^{1, p}(\Omega)\right)$ as follows:

$$
\bar{\phi}(u)= \begin{cases}u & \text { if }\|u\|>\rho, \\ \frac{1}{\eta_{k}} \sqrt{\rho^{2}-\|u\|^{2}} \psi_{k}+u & \text { if }\|u\| \leq \rho .\end{cases}
$$


We notice that $\bar{\phi}$ satisfies the required assumptions; indeed if $u \in V_{k-1}^{\sigma}$ and $\|u\|>\rho$, then by (4.10) it is $J(\bar{\phi}(u))=J(u) \leq-\delta_{1} \rho^{p}<0$; on the other hand, if $u \in V_{k-1}^{\sigma}$ and $\|u\| \leq \rho$, then by (4.8) it results $\bar{\phi}(u) \in V^{\sigma}$; moreover direct computations imply that $\|\bar{\phi}(u)\| \leq \sqrt{2} \rho$, while by (2.2) we obtain $\|\bar{\phi}(u)\| \geq \frac{\rho}{L}$, thus by (4.13) it follows $J(\bar{\phi}(u)) \leq-\delta_{2}$. Summing up, (4.12) holds, so $c<0$ and the proof is complete.

Proof of Theorem 1.5. By Proposition 3.1(ii) the functional $J$ satisfies (CPS) and we can proceed as in the proof of Theorem 1.4.

Remark 4.1. Existence results still hold if the limit in assumption $\left(H_{2}\right)$ is infinite. More precisely, if the assumptions $\left(H_{0}\right),\left(H_{1}\right)$ and $\left(H_{3}\right)$ hold and moreover we assume $\left(H_{2}^{\prime}\right)$ and $\left(H_{4}^{\prime}\right)$ as in Remark 3.2 , then $\left(P_{\infty}\right)$ has at least a nontrivial solution. Indeed, by Proposition 3.1, arguments similar to those in the proof of Theorem 1.1 show that we can apply the Mountain Pass Theorem (cf. [21, Theorem 2.2]), as by $\left(H_{2}^{\prime}\right)$ it follows $J(u) \geq \beta>0$ for any $u \in S_{\rho}, \rho>0$, and by $\left(H_{4}^{\prime}\right)$, for a suitable $k$-dimensional $V_{k}^{\sigma}, J$ tends to $-\infty$ as $\|u\|$ diverges on $V_{k}^{\sigma}$, getting the existence of a solution corresponding to a critical level $c$ such that $c \geq \beta>0$.

On the other hand, if $\left(H_{2}\right)$ and $\left(H_{4}\right)$ are replaced by $\left(H_{2}^{\prime \prime}\right)$, then fixing $k \in \mathbb{N}$ such that $\eta_{k}>l_{\infty}$ (recall that $\left(\eta_{k}\right)_{k}$ diverges), there exists $\beta<0$ such that $J(u) \geq \beta$ for all $u \in W_{k-1}$. Moreover, for any finite dimensional subspace $V$ and any $\rho>0$, it results $J(u) \leq \alpha$ for all $u \in V \cap S_{\rho}$ for a suitable $\alpha<0$, hence by Example 2.2, Proposition 3.1 and Theorem 2.4 applied to $S=W_{k-1}$ and $Q=V_{k-1} \cap B_{\rho}$ (see $(2.3)$ ), we get the existence of a critical level $c$, which a priori could be 0 , since $\sup _{u \in Q} J(u)=0$. But, considering again $\bar{\phi}$ as in the proof of Theorem 1.4, we get that (4.12) holds, thus $c<0$ and $\left(P_{\infty}\right)$ has at least a non-trivial solution.

Remark 4.2. The arguments in Remark 4.1 still work in the resonant case.

\section{References}

[1] Amann, H., Zehnder, E.: Nontrivial solutions for a class of nonresonance problems and applications to nonlinear differential equations. Ann. Scuola Norm. Sup. Pisa Cl. Sci. 7(4), 539-603 (1980)

[2] Anane, A.: Etude des valeurs propres et de la résonance pour l'opérateur p-laplacien. Thèse de Doctorat, Université Libre de Bruxelles (1987)

[3] Anane, A., Gossez, J.P.: Strongly nonlinear elliptic problems near resonance: a variational approach. Comm. Partial Differ. Equ. 15, 1141-1159 (1990)

[4] Arcoya, D., Orsina, L.: Landesman-Lazer conditions and quasilinear elliptic equations. Nonlinear Anal. 28, 1623-1632 (1997) 
[5] Bartolo, P., Benci, V., Fortunato, D.: Abstract critical point theorems and applications to some nonlinear problems with "strong" resonance at infinity. Nonlinear Anal. 7, 981-1012 (1983)

[6] Bartolo, R., Candela, A.M., Salvatore, A.: Perturbed asymptotically linear problems. Ann. Mat. Pura Appl. (to appear). doi:10.1007/s10231-012-0267-9

[7] Benci, V.: On the critical point theory for indefinite functionals in the presence of symmetries. Trans. Am. Math. Soc. 274, 533-572 (1982)

[8] Brezis, H.: Functional Analysis, Sobolev Spaces and Partial Differential Equations. Universitext XIV, Springer, New York (2011)

[9] Costa, D.G., Magalhães, C.A.: Existence results for perturbations of the $p$-Laplacian. Nonlinear Anal. 24, 409-418 (1995)

[10] Candela, A.M., Palmieri, G.: Infinitely many solutions of some nonlinear variational equations. Calc. Var. 34, 495-530 (2009)

[11] Dinca, G., Jebelean, P., Mawhin, J.: Variational and topological methods for Dirichlet problems with $p$-Laplacian. Port. Math. 58, 339-378 (2001)

[12] Drábek, P., Robinson, S.: Resonance problems for the $p$-Laplacian. J. Funct. Anal. 169, 189-200 (1999)

[13] Fadell, E.R., Rabinowitz, P.H.: Generalized cohomological index theories for Lie group actions with an application to bifurcation questions for Hamiltonian systems. Invent. Math. 45, 139-174 (1978)

[14] García Azorero, J., Peral Alonso, I.: Existence and nonuniqueness for the p-Laplacian: Nonlinear eigenvalues. Comm. Partial Differ. Equ. 12, 1389 1430 (1987)

[15] Li, G., Zhou, H.S.: Asymptotically linear Dirichlet problem for the $p$-Laplacian. Nonlinear Anal. 43, 1043-1055 (2001)

[16] Li, G., Zhou, H.S.: Multiple solutions to $p$-Laplacian problems with asymptotic nonlinearity as $u^{p-1}$ at infinity. J. Lond. Math. Soc. 65, 123-138 (2002)

[17] Lindqvist, P.: On the equation $\operatorname{div}\left(|\nabla u|^{p-2} \nabla u\right)+\lambda|u|^{p-2} u=0$. Proc. Am. Math. Soc. 109, 157-164 (1990)

[18] Lindqvist, P.: On a nonlinear eigenvalue problem. Berichte Univ. Jyväskylä Math. Inst. 68, 33-54 (1995)

[19] Liu, S., Li, S.: Existence of solutions for asymptotically 'linear' p-Laplacian equations. Bull. Lond. Math. Soc. 36, 81-87 (2004)

[20] Perera, K., Szulkin, A.: p-Laplacian problems where the nonlinearity crosses an eigenvalue. Discr. Contin. Dyn. Syst. 13, 743-753 (2005)

[21] Rabinowitz, P.H.: Minimax Methods in Critical Point Theory with Applications to Differential Equations. CBMS Regional Conference Series in Mathematics 65, American Mathematical Society, Providence (1986) 
[22] Rabinowitz, P.H.: Variational Methods for Nonlinear Eigenvalues Problems, (G. Prodi Ed.), Edizioni Cremonese, Roma, pp. 141-195 (1974)

[23] Struwe, M.: Variational Methods. Applications to Nonlinear Partial Differential Equations and Hamiltonian Systems. 4rd Edition, Ergeb. Math. Grenzgeb. (4) 34, Springer, Berlin (2008)

[24] Willem, M.: Minimax Theorems. Birkhäuser, Boston (1996)

Rossella Bartolo

Dipartimento di Meccanica, Matematica e Management

Politecnico di Bari

Via E. Orabona 4

70125 Bari

Italy

e-mail: r.bartolo@poliba.it

Anna Maria Candela and Addolorata Salvatore

Dipartimento di Matematica

Università degli Studi di Bari "Aldo Moro"

Via E. Orabona 4

70125 Bari

Italy

e-mail: annamaria.candela@uniba.it

Addolorata Salvatore

e-mail: addolorata.salvatore@uniba.it

Received: 22 December 2012.

Accepted: 26 February 2013. 